\title{
The burden of new onset atrial fibrillation in the intensive care unit (ICU). an observational, prospective, single-center study
}

\author{
A Papathanasiou, E Roustanis, X Zikou, E Galiatsou, G Papathanakos, G Zilis, M Saranti, V Koulouras, A Karachaliou, \\ G Nakos ${ }^{*}$
}

From ESICM LIVES 2015

Berlin, Germany. 3-7 October 2015

\section{Intr}

Cardiac arrhythmias constitute a great challenge in the $\mathrm{ICU}$, since they aggravate a borderline cardiopulmonary function. Atrial fibrillation (AF) forms the commonest type of arrhythmias and an unappreciated chapter of the respective literature at the same time.

\section{Objectives}

To estimate the prevalence of new onset AF in ICU patients.

\section{Methods}

the study took place in a 13 level-3 bed ICU, of a university hospital in Northwestern Greece, during December 2013-September 2014. All admitted patients were scrutinized and their medical history, APACHE II score, admission and discharge diagnosis, AF prevalence along with arterial blood gases (ABGs) and vital signs during the attack, arrhythmia's treatment outcome and any possible damaging factors responsible for its appearance were recorded.

\section{Results}

A total of 192 patients were admitted during the study period (124 men, 68 women, mean age 59.2 and 59.8 years respectively). Total mortality was $27.6 \%$, mean ICU staying was 15 days (1-77 days). Twelve patients had history of chronic AF (CAF) and 7 had history of paroxysmic AF (PAF). During hospitalization, 37 patients developed rapid ventricular response AF (RVAF, 7/12-58.33\% with CAF, 4/7-57.14\% with PAF and 18/173-10.4\% with no history of arrhythmias). Main factors for RVAF, common in all three

University Hospital of Ioannina, Dept of ICU, Ioannina, Greece groups, were hypoxemia, sepsis and septic shock, severe electrolyte abnormalities and APACHE II score above 30 . Amiodarone (300 mg in 30 minute infusion, followed by either $900 \mathrm{mg}$ in 24-hour infusion or $300 \mathrm{mg}$ tid) was the drug of choice in $32 / 37$ patients, while in $5 / 37$ patients (all with CAF and RVAF), iv b-blocker was initially administered. In 4/5 with CAF and RVAF, b-blocker was ineffective and amiodarone was additionally administered. In all patients with CAF and RVAF, amiodarone was effective for rate control. Amiodarone was also the drug of choice for rhythm restoration in all RVAF and history of PAF and those with no history of arrhythmias. Restoration of rhythm was successful in all of those patients. In all cases, RVAF improved quicker when the underlying condition was hypoxemia, rather than sepsis or septic shock, while electrolyte abnormalities rendered RVAF especially resistant to treatment, until those abnormalities were effectively addressed. Moreover, CAF patients were proven more difficult to respond to drugs than PAF ones. In most cases, initial $300 \mathrm{mg}$ of amiodarone sufficed for RVAF control.

\section{Conclusions}

RVAF is frequent in ICU patients, especially among those with prior history of cardiac disease. Proper treatment requires immediate control of the predisposing factor and drug regimen initiation, mainly amiodarone.

Published: 1 October 2015

doi:10.1186/2197-425X-3-S1-A212

Cite this article as: Papathanasiou et al:: The burden of new onset atrial fibrillation in the intensive care unit (ICU). an observational, prospective, single-center study. Intensive Care Medicine Experimental 2015 3(Suppl 1): A212. 